\title{
Classification of Solitons for the Affine Curvature Flow
}

\author{
Levi Lopes de Lima AND José Fabio Montenegro ${ }^{1}$
}

\begin{abstract}
The curvature flow in planar affine geometry is introduced and the classification of solitons (homothetic solutions) for this flow is carried out via the method of adjoint orbits. This technique is used to integrate, up to a single quadrature, the soliton equations in terms of elliptic functions. In particular, it is shown that ellipses are the only embedded solitons.
\end{abstract}

\section{Introduction.}

In recent years there has been much interest in investigating the asymptotic behavior of curves in the euclidean plane $\mathbf{R}^{2}$ evolving according to a flow of the type

$$
\mathbf{X}_{t}=\mathbf{W}
$$

Here, $\mathbf{X}=\mathbf{X}(t, u):[0, T) \times I \rightarrow \mathbf{R}^{2}$ is a one-parameter family of closed curves and $\mathbf{W}$ is a vector field defined in some neighborhood of $\mathbf{X}_{0}=\mathbf{X}(0,$.$) .$ Usually, $\mathbf{W}$ is the gradient vector field of some geometric functional $\mathcal{E}$ defined in the space of curves $\mathbf{X}: I \rightarrow \mathbf{R}^{2}$. Flows studied so far include:

- $\mathcal{E}(\mathbf{X})=$ length of $(\mathbf{X})$. This is the curve shortening flow ([AL], [GH], [G]). Some generalizations of this flow have also deserved attention ([GL],[A1]).

- $\mathcal{E}(\mathbf{X})=$ total squared curvature of $\mathbf{X}$. This is the curve straightening flow $([\mathrm{LS}],[\mathrm{L}])$.

The general strategy to understand these flows can be organized in the following steps: (i) classification of solitons (homothetic solutions); (ii) linear stability analysis around the solitons; (iii) local and global existence of

\footnotetext{
${ }^{1}$ Both authors have been partially supported by FUNCAP/CE.
} 
solutions (possibly with some restrictive condition on the initial data); (iv) asymptotic behavior of solutions and corresponding estimates on the basins of attraction of solitons.

The purpose of this paper is to add a new flow to this list and carry out the first step in the program described above. So we consider the flow of planar curves by (unimodular) affine curvature. More precisely, let $\mathcal{G} \subset$ $G L(3, \mathbf{R})$ be the group of real matrices of the type

$$
\left(\begin{array}{lll}
1 & 0 & 0 \\
e & a & b \\
f & c & d
\end{array}\right)
$$

where the entries satisfy $a d-b c=1$. Now, the usual action of $G L(3, \mathbf{R})$ on $\mathbf{R}^{\mathbf{3}}$ induces an action of $\mathcal{G}$ on $\mathbf{R}^{2}$ by affine motions. This is given explicitly by

$$
\mathbf{x} \mapsto \mathcal{A} \mathbf{x}+\mathbf{b},
$$

where we identify $\mathbf{x}=(x, y)^{T}$ to $(1, x, y)^{T}, \mathbf{b}=(e, f)^{T}$ and

$$
\mathcal{A}=\left(\begin{array}{ll}
a & b \\
c & d
\end{array}\right) .
$$

The affine geometry of curves is then the study of properties of curves invariant under this action. For locally strictly convex curves $\mathbf{X}: I \rightarrow \mathbf{R}^{2}$, a satisfactory theory is available (see [Bl] and sections 2 and 3 below for more precise statements) and we briefly review some of it now (in what follows, given vectors $\mathbf{a}, \mathbf{b} \in \mathbf{R}^{2}$, we denote by $(\mathbf{a}, \mathbf{b})$ the $2 \times 2$-matrix whose columns are $\mathbf{a}$ and $\mathbf{b}$ in this order and by $|\mathbf{a}, \mathbf{b}|:=\operatorname{det}(\mathbf{a}, \mathbf{b})$ the skew-symmetric pairing given by the determinant):

I) After rewriting $\mathbf{X}=\mathbf{X}(s)$, where $s$ is the affine arclength parameter, a natural frame $(\mathbf{T}, \mathbf{N}) \in S L(2, \mathbf{R})$ is defined over $\mathbf{X}$. Here, $\mathbf{T}=\mathbf{X}_{s}$ and $\mathbf{N}=\mathbf{X}_{s s}$ are the affine tangent and affine normal to $\mathbf{X}$, respectively. The affine curvature $k=\left|\mathbf{X}_{s s}, \mathbf{X}_{s s s}\right|$ shows up in the structure equation $\mathbf{N}_{s}=-k \mathbf{T}$ and determines $\mathbf{X}$ up to affine motions;

II) Conics are the curves with constant affine curvature, so that parabolae (ellipses, hyperbolae, resp.) have curvature zero (greater than zero, lesser than zero, resp.);

III) The affine curvature $k$ has a nice variational interpretation. More precisely, if $\mathbf{X}(t,),. t \in[0, \epsilon)$, satisfies $\mathbf{X}_{t}=f \mathbf{N}$ then, under suitable 
boundary conditions, the affine length $l$ satisfies

$$
l^{\prime}(0)=-\frac{2}{3} \int f k d s,
$$

a formula already known to Blaschke ([Bl]). In particular, arcs of parabolae are extremals for $l$.

At this juncture, in analogy with the curve shortening problem, it seems natural to investigate the flow $\mathbf{X}_{t}=k \mathbf{N}$. It turns out, however, that this is not the most natural flow to consider here and the reason for this is quite surprising: in affine geometry curves tend to maximize their affine length (see comments following (3.9) below). This leads us then, in view of (1.1), to study the flow

$$
\mathbf{X}_{t}=-k \mathbf{N}
$$

which we call the affine curvature flow.

In this paper we give a complete classification of solitons for this flow. Our approach is inspired by the classification of solitons for the curve shortening flow given by Abresch and Langer [AL] and consists of renormalizing the flow by adding a tangent component $g \mathbf{T}$ to the velocity vector field $\mathbf{W}=-k \mathbf{N}$ so that each $\mathbf{X}(t,$.$) now has constant affine speed. This enables$ one to compute the evolution equation satisfied by the renormalized curvature $\kappa=\xi^{2} k$, where $\xi$ is the affine speed. It turns out that this is a fourth order nonlinear parabolic equation. Nevertheless, the soliton condition implies a remarkable cancellation in this equation and thus classification can be carried out. More precisely, solitons are shown to correspond to periodic curvature functions $\kappa$ satisfying the second order ordinary differential equation

$$
\ddot{\kappa}+\kappa^{2}-1=0
$$

and generating closed curves via the structure equations. Our main result is then the following:

Theorem 1.1 (Classification of solitons in affine geometry). Let $\mathrm{X}$ be a soliton for the affine curvature flow (1.2). Then we have the following possibilities for $\mathbf{X}$ :

i) $\mathbf{X}$ is an ellipse (this corresponds to $\kappa \equiv 1$, which obviously solves (1.3)); 
ii) $\mathbf{X}=\mathbf{X}_{p, q}$, a curve whose affine curvature $\kappa_{p, q}$ is a nonconstant periodic solution of (1.3) depending upon $(p, q) \in \mathbf{N} \times \mathbf{N}$. The pair $(p, q)$ is by no means arbitrary and must be such that $p / q$ is confined to the open interval $(1 / 2, \sqrt{2} / 2)$.

Remark 1.1. It follows from classification that $\mathbf{X}_{p, q}$ has $2 q$ sextactic points (points where $\dot{\kappa}_{p, q}=0$ ) and winding number $p$ (see fig. 2, where some curves are displayed). In particular, ellipses are the only embedded solitons.

Remark 1.2. We note that our classification is, from a qualitative viewpoint, entirely similar to the one for solitons in the curve shortening flow ([AL])as well as to the one given for elasticae in the hyperbolic plane ([BG],[LS]) in the sense that in all of these flows, the same arithmetic condition singles out the closed solutions. It is a quite surprising fact that classification of closed solutions for such a diverse class of problems ends up in the same numerical criterion.

Remark 1.3. We should mention that the rationality condition in the theorem above expresses the fact that there exist periodic solutions of (1.3) (in fact, an uncountable number of them corresponding to irrational numbers in $(1 / 2, \sqrt{2} / 2))$ generating bounded curves which never close up and, hence, do not define a soliton. In fact, a much more interesting picture emerges from our classification: there do exist periodic solutions of (1.3) which generate unbounded curves (see fig. 3, where some of these curves are displayed). As we shall see, this happens if and only if the curvature assumes some nonpositive value and, moreover, the set of such curves, up to affine motions, is parametrized by a bounded semi-open interval.

Remark 1.4. Regarding steps (iii) and (iv) mentioned above, one should refer to recent work of $\mathrm{B}$. Andrews ([A2]), where it is shown that the motion of any smooth embedded closed convex plane curve under (1.2) can be continued smoothly for all time, the curve remaining strictly convex while expanding to infinite size and approaching a homothetically expanding ellipse. We remark that a crucial step in his analysis is the use of the uniqueness assertion about ellipses in Remark 1.1 above.

This paper is organized as follows. In section 2, we recall the relevant definitions and facts in plane affine geometry. In section 3 , we derive the first variation formula and introduce the affine curvature flow. In section 4 , we adapt the Abresch-Langer approach to our setting so that the flow gets 
renormalized and, as a consequence, the evolution equations for the renormalized affine curvature are presented. In section 6 , soliton solutions for this flow are considered and in, section 7, the analysis leading to the classification theorem above is carried out. A crucial ingredient in this analysis is the method of adjoint orbits [BG]. This technique enables one to integrate the structure equations and determine the parametrization of solitons explicitly up to a single quadrature in terms of elliptic functions. The paper also contains an appendix where two different definitions of soliton solutions are shown to be equivalent.

Acknowledgements. The authors would like to express their deep gratitude to R. L. Bryant for sharing with them his expertise on conservation laws and specially for bringing the method of adjoints orbits to their attention. Special thanks are also due to $M$. do Carmo for enlightening conversations on affine geometry, to R. Iório Jr. for discussions on the analytical well-posedness of (4.9) below and to the referee for helpful comments and suggestions.

\section{Preliminaries on Affine Geometry.}

We shall consider planar regular curves $\mathbf{X}=\mathbf{X}(u)=(x(u), y(u)), u \in I \subset$ R. From now on, we shall assume that the quantity

$$
\alpha=\left|\mathbf{X}_{u}, \mathbf{X}_{u u}\right|
$$

is strictly positive. This means, of course, that $\mathbf{X}$ is locally strictly convex and reflects the well-known fact that curves with inflection points cannot be directly treated in affine geometry.

In order to study the affine geometry of $\mathbf{X}$ we introduce the frame

$$
u \in I \longmapsto \mathbf{g}=(\mathbf{T}, \mathbf{N}) \in S L(2, \mathbf{R})
$$

over $\mathbf{X}$, where $\mathbf{T}=\mathbf{X}_{u} / \sqrt{\alpha}$ and $\mathbf{N}=\mathbf{X}_{u u} / \sqrt{\alpha}$. A straightforward computation yields

$$
\mathbf{g}^{-1} \mathbf{g}_{u}=\left(\begin{array}{cc}
-\alpha_{u} / 2 \alpha & -\gamma / \alpha \\
1 & \alpha_{u} / 2 \alpha
\end{array}\right), \quad \gamma=\left|\mathbf{X}_{u u}, \mathbf{X}_{u u u}\right| .
$$

Now, we seek a change of parameter $s=s(u)$ such that $\alpha \equiv 1$ relative to $s$. If this is the case one should have

$$
1=\left|\mathbf{X}_{s}, \mathbf{X}_{s s}\right|=\left|\mathbf{X}_{u} \frac{d u}{d s}, \mathbf{X}_{u u}\left(\frac{d u}{d s}\right)^{2}+\mathbf{X}_{u} \frac{d^{2} u}{d s^{2}}\right|=\alpha\left(\frac{d u}{d s}\right)^{3}
$$


and it suffices to set $s=\int \xi d u, \xi=\alpha^{1 / 3}$. Here, $\xi$ is the affine speed of $\mathbf{X}$ (relative to $u$ ) and $s$ is the affine arclength parameter. The affine tangent and affine normal to $\mathbf{X}$ are $\mathbf{T}=\mathbf{X}_{s}$ and $\mathbf{N}=\mathbf{X}_{s s}$, respectively. Observe that $\mathbf{g}=(\mathbf{T}, \mathbf{N}) \in S L(2, \mathbf{R})$. The structure equations in affine geometry now read

$$
\left\{\begin{array}{l}
\mathbf{X}_{s}=\mathbf{T} \\
\mathbf{T}_{s}=\mathbf{N} \\
\mathbf{N}_{s}=-k \mathbf{T}
\end{array}\right.
$$

where

$$
k=\left|\mathbf{X}_{s s}, \mathbf{X}_{s s s}\right|
$$

is the affine curvature of $\mathbf{X}$.

Integration of (2.3) shows that $k$ determines $\mathbf{X}$ up to affine motions (see [Bl] for details). In particular, the important special case $k=$ const. is easily handled, yielding ellipses $(k>0)$, parabolae $(k=0)$ and hyperbolae $(k<0)$. For example, if $\mathbf{X}(u)=(a \cos u, b \sin u), 0 \leq u \leq 2 \pi$, is the usual parametrization of the ellipse with semi-axes $a$ e $b$, we get $\xi=(a b)^{1 / 3}$, $s=(a b)^{1 / 3} u$ and $k=(a b)^{-2 / 3}$. In particular, two ellipses have the same affine curvature if and only if they bound the same area.

\section{First Variational Formula and the Affine Curvature Flow.}

By the previous section, the affine length of a curve $\mathbf{X}:[0,1] \rightarrow \mathbf{R}^{\mathbf{2}}$ is given by

$$
l:=\int_{0}^{1} \xi d u, \quad \xi=\left|\mathbf{X}_{u}, \mathbf{X}_{u u}\right|^{1 / 3} .
$$

We now compute how the functional $l$ varies when $\mathbf{X}$ is smoothly deformed.

More precisely, let $\mathbf{X}=\mathbf{X}(t, u), 0 \leq t<\epsilon, 0 \leq u \leq 1$, be a smooth variation of $\mathbf{X}_{0}=\mathbf{X}(0,$.$) such that$

$$
\mathbf{X}_{t}=f \mathbf{N}+g \mathbf{T} .
$$

Here, $f$ and $g$ are arbitrary smooth functions of $t$ and $u$. We remark that the factor $g \mathbf{T}$ can be chosen at our convenience since it only amounts to a reparametrization of $\mathbf{X}$. We consider the affine length functional

$$
l(t)=\int_{0}^{1} \xi d u
$$


and wish to compute

$$
l^{\prime}(t)=\int_{0}^{1} \xi_{t} d u
$$

In order to determine $\xi_{t}$, we start with the identities

$$
\mathbf{X}_{u}=\xi \mathbf{T}, \quad \mathbf{T}_{u}=\xi \mathbf{N}, \quad \mathbf{N}_{u}=-\xi k \mathbf{T},
$$

which follow from the structure equations (2.3). Taking the derivative of both sides of $\mathbf{X}_{u}=\xi \mathbf{T}$ with respect to $t$ and using (3.3) we get

$$
\xi_{t} \mathbf{T}+\xi \mathbf{T}_{t}=\left(g_{u}-\xi k f\right) \mathbf{T}+\left(f_{u}+\xi g\right) \mathbf{N} .
$$

Pairing this equation with $\mathbf{T}$ and $\mathbf{N}$, we obtain successively

$$
\begin{aligned}
& \left|\mathbf{T}_{t}, \mathbf{T}\right|=-\xi^{-1}\left(f_{u}+\xi g\right), \\
& \xi_{t}+\xi\left|\mathbf{T}_{t}, \mathbf{N}\right|=g_{u}-\xi k f .
\end{aligned}
$$

Taking the derivative of (3.5) with respect to $u$, then using (3.3) and the fact that $|\mathbf{T}, \mathbf{N}|=1$ implies $\left|\mathbf{N}_{t}, \mathbf{T}\right|=\left|\mathbf{T}_{t}, \mathbf{N}\right|$, we have

$$
-\left(\xi^{-1}\left(f_{u}+\xi g\right)\right)_{u}=-\xi_{t}+2 \xi\left|\mathbf{T}_{t}, \mathbf{N}\right|
$$

Now , (3.6) and (3.7) imply the desired formula for $\xi_{t}$ :

$$
\xi_{t}=-\frac{2}{3} \xi k f+\frac{2}{3} g_{u}+\frac{1}{3}\left(\xi^{-1}\left(f_{u}+\xi g\right)\right)_{u}
$$

If either every curve in the variation is closed or if $f_{u}(0)=f_{u}(1)=g(0)=$ $g(1)=0$ (clamped variations), we retrieve the first variation formula

$$
l^{\prime}(t)=-\frac{2}{3} \int_{0}^{1} k f d s,
$$

furnishing a variational interpretation for $k$.

In particular, (3.9) implies that if we are given two points $P$ and $Q$ in the plane and two concurrent straight lines $m$ and $n$ passing through $P$ and $Q$ respectively, then the unique arc of parabola determined by this configuration is an extremal for $l$ along variations by locally convex curves passing through $P$ and $Q$ and tangent to $m$ and $n$ at these points.

It is an amazing fact, however, that curves in affine geometry are compelled to maximize their affine length. In fact, it is not hard to check that 
the arcs of parabolae referred to above are global maximizers for $l$ under clamped variations (see $[\mathrm{Bl}]$ and remark 4.2 below). Taking into account (3.9), this is the reason why, as mentioned in the Introduction, the natural flow to study in affine geometry is

$$
\mathbf{X}_{t}=-k \mathbf{N} .
$$

We call this the affine curvature flow.

\section{Renormalizing the Affine Curvature Flow.}

We return now to the general evolution equation (3.1). As we noticed above, the tangential component $g \mathbf{T}$ can be chosen at our convenience since it clearly just amounts to a reparametrization of $\mathbf{X}$. We take advantage of this fact and, inspired by [AL], we shall renormalize the flow (3.1) by choosing $g$ such that evolution by

$$
\mathbf{X}_{t}=f \mathbf{N}+g \mathbf{T}
$$

goes through curves with constant affine speed. The starting point is (3.8), which we rewrite as

$$
\frac{3}{2}(\ln \xi)_{t}=-k f+\frac{g_{u}}{\xi}+\frac{1}{2} \frac{\left(\xi^{-1}\left(f_{u}+\xi g\right)\right)_{u}}{\xi} .
$$

Assuming that every $\mathbf{X}(t,$.$) is closed, one easily gets$

Proposition 4.1. If $\mathbf{X}$ evolves according to (4.1) then the following sentences are equivalent:

(i) Each $\mathbf{X}(t,$.$) has constant affine speed;$

(ii) $\mathbf{X}(0,$.$) has constant affine speed and$

$$
\frac{3}{2} \frac{g_{u}}{\xi}=-\frac{1}{2} \frac{f_{u u}}{\xi^{2}}+k f-\int_{0}^{1} k f d u
$$

(iii) $\xi_{u} \equiv 0$

From now on, we shall consider closed curves $\mathbf{X}(t,$.$) evolving according$ to the evolution law

$$
\mathbf{X}_{t}=f \mathbf{N}+g \mathbf{T},
$$


where $g$ is determined by the proposition above. Clearly, $g$ depends on $f$ and in the case that $f=-k$, we call the corresponding evolution law

$$
\mathbf{X}_{t}=-k \mathbf{N}+g \mathbf{T}
$$

the renormalized affine curvature flow.

The proposition above is the starting point for the derivation of the evolution equation for the renormalized affine curvature $\kappa$. First, (4.3) implies that the $\mathbf{T}$-component of $\mathbf{X}_{\text {tuuu }}$ is

$$
\left(\left(\left(g_{u}-\xi k f\right)_{u}-\xi k\left(f_{u}+\xi g\right)\right)_{u}-\xi k\left(\xi\left(g_{u}-\xi k f\right)+\left(f_{u}+\xi g\right)_{u}\right)\right) \mathbf{T} .
$$

On the other hand, since, by Proposition 4.1, $\xi_{u}=0$, (3.3) implies $X_{u u u}=$ $-\xi^{3} k \mathbf{T}$. Hence,

$$
\mathbf{X}_{t u u u}=-3 \xi^{2} \xi_{t} k \mathbf{T}-\xi^{3} k_{t} \mathbf{T}-\xi^{3} k \mathbf{T}_{t} .
$$

Pairing (4.5) and (4.6) with $\mathbf{N}$ we obtain

$$
\begin{aligned}
3 \xi^{2} k \xi_{t}+\xi^{3} k_{t}+\xi^{3} k\left|\mathbf{T}_{t}, \mathbf{N}\right|= & \xi k\left(\xi\left(g_{u}-\xi k f\right)+\left(f_{u}+\xi g\right)_{u}\right) \\
& -\left(\left(g_{u}-\xi k f\right)_{u}-\xi k\left(f_{u}+\xi g\right)\right)_{u} .
\end{aligned}
$$

Using (3.6) this can be written as

$$
\xi\left(\xi^{2} k\right)_{t}=\xi k\left(f_{u}+\xi g\right)_{u}-\left(\left(g_{u}-\xi k f\right)_{u}-\xi k\left(f_{u}+\xi g\right)\right)_{u}
$$

and, after straightforward simplifications, as

$$
\xi\left(\xi^{2} k\right)_{t}=3 \xi k f^{\prime \prime}+3 \xi k^{\prime} f^{\prime}+\xi k^{\prime \prime} f+2 \xi^{2} k g^{\prime}+\xi^{2} k^{\prime} g-g^{\prime \prime \prime},
$$

where primes denote derivation with respect to $u$.

Now, if we consider the evolution of a closed curve under (4.4) then, by defining renormalized quantities

$$
\kappa=\xi^{2} k, \quad h=\xi^{3} g, \quad d t / d \tau=\xi^{4},
$$

we obtain from (4.2) and (4.7) with $f=-k$

$$
\left\{\begin{array}{l}
\kappa_{\tau}=-4 \kappa \kappa^{\prime \prime}-3 \kappa^{2}+2 \kappa h^{\prime}+\kappa^{\prime} h-h^{\prime \prime \prime} \\
\frac{3}{2} h^{\prime}=\frac{1}{2} \kappa^{\prime \prime}-\kappa^{2}+\int_{0}^{1} \kappa^{2} d u
\end{array}\right.
$$

The above system then describes how the renormalized affine curvature $\kappa$ evolves under the renormalized affine curvature flow (4.4). 
Remark 4.1. Notice that the second equation in (4.9) only determines $h$ up to a additive constant and this can in principle affect the evolution of $\kappa$ given by the first equation. We see, however, that $h \mathbf{T}$ is a tangential term added to the geometric velocity $-\kappa \mathbf{N}$ and as such it does not affect the geometry of the evolution in the sense that if we replace $h$ by $h$ plus a constant, the resulting evolution gets unchanged geometrically. In other words, after solving (4.9) for $\kappa$ and then solving the structure equation (2.3) for $\mathbf{X}$ we end up with the same curves up to tangential reparametrizations.

Remark 4.2. Formula (4.7) above also can be used to compute the second variation formula for arcs of parabolae. The calculation implies that these arcs are local maximizers for $l$ and furnishes further evidence for the choice of sign made above, but we shall not pursue this here.

Remark 4.3. The system (4.9) can be rewritten as a single fourth order parabolic equation, namely,

$$
\kappa_{\tau}=-\frac{1}{3} \kappa^{\prime \prime \prime \prime}+\text { lower order nonlinear terms, }
$$

and this accounts for the difficulty of analysing the affine curvature flow for arbitrary initial data. In particular, a crucial ingredient in the analysis of the asymptotic behaviour of second order flows, namely, the parabolic maximum principle, is not available here. Despite this difficulty, this equation has been recently successfully analysed by B. Andrews ([A2]) by using integral estimates (see Remark 1.4 above.) We notice here that the system (4.9) is locally well-posed precisely because of the minus sign in the linear part of (4.10).

\section{The Soliton Equations: solving for $\kappa$.}

We now look for a special class of solutions of (4.9). We start with a

Definition 5.1. A soliton for the affine curvature flow (1.2) is a solution $\mathbf{X}=\mathbf{X}(t,),. t \in\left[0, t_{0}\right)$, by closed curves satisfying $\kappa_{\tau}=0$ and $\left|\mathbf{T}, \mathbf{T}_{t}\right|=0$.

Since the renormalized curvature $\kappa$ is clearly invariant under homotheties, the first condition requires that the homothety type be preserved along the evolution. We show in the Appendix that solitons solutions as defined here correspond precisely to solution of (1.2) which evolve by dilation about some point. On the other hand, it follows from (3.5) with $f=-k$ that the second condition is equivalent to $h=\kappa^{\prime}$. In other words, we are just 
prescribing the tangential velocity of solitons as they evolve. Inserting this information into (4.9) one gets

$$
\left\{\begin{array}{l}
\kappa^{\prime \prime \prime \prime}+2\left(\kappa^{\prime}\right)^{2}+2 \kappa \kappa^{\prime \prime}=0 \\
\kappa^{\prime \prime}+\kappa^{2}-\mu=0
\end{array}\right.
$$

but clearly the first equation equals the second derivative of the second one and we conclude that solitons correspond to periodic solutions of

$$
\kappa^{\prime \prime}+\kappa^{2}-\mu=0, \quad \mu=\int_{0}^{1} \kappa^{2} d u
$$

generating closed curves via the structure equations (2.3). This is the problem we now start to analyse.

Without loss of generality, one can make $\mu=1$ in (5.2). In fact, one can easily check that this amounts to replacing $\kappa(s)$ by $\mu^{-1 / 2} \kappa\left(\mu^{-1 / 4} s\right)$ and this just reflects the possibility of rescaling the curve. So we now look for solutions in $\mathbf{X}$ for the system

$$
\dot{\mathbf{X}}=\mathbf{T}, \quad \dot{\mathbf{T}}=\mathbf{N}, \quad \dot{\mathbf{N}}=-\kappa \mathbf{T},
$$

where $\kappa: \mathbf{R} \rightarrow \mathbf{R}$ is a periodic solution to the equation

$$
\ddot{\kappa}+\kappa^{2}-1=0 .
$$

From now on, dots will denote derivation with respect to affine arclength.

More precisely, we wish to determine for which periodic solutions of (5.4) the system (5.3) has a closed solution in $\mathbf{X}$. In the next section, we will be able to analyse this system for any periodic curvature solution by using the method of adjoint orbits. As an outcome of this, we can determine precisely, in terms of a certain elliptic integral, which solutions $\mathbf{X}$ close up and this yields the numerical condition in the classification theorem.

For the time being, however, we indicate how periodic solutions of (5.4) can be expressed in terms of elliptic functions. First observe that the 'energy'

$$
E=\dot{\kappa}^{2}+\frac{2}{3} \kappa^{3}-2 \kappa
$$

is a first integral for (5.4). The phase portrait of (5.5) in the plane $(\kappa, \dot{\kappa})$ can be easily determined and is depicted below. 


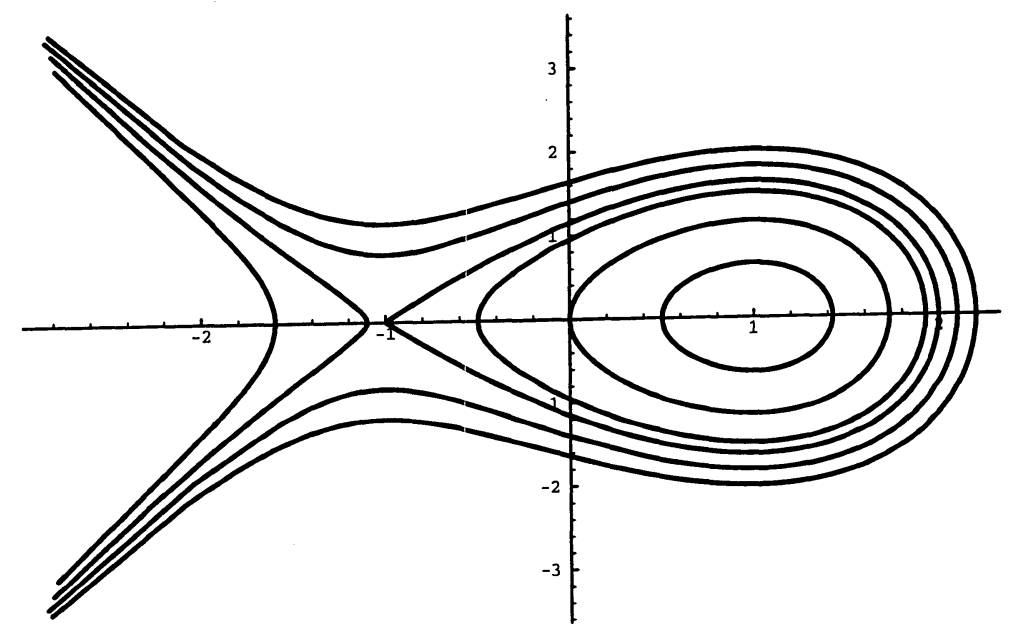

Figure 1: Phase Portrait

We now analyse the periodic solutions of (5.4). Clearly, $\kappa=1$ corresponds to an ellipse (which is obviously a soliton) and $\kappa=-1$ generates a hyperbola, which is not closed, hence not a soliton. As for the nontrivial solutions of (5.4), we see that they are periodic if and only if $-4 / 3<E<4 / 3$, and we can classify them according to the minimum value $\kappa_{\min }$ of $\kappa$, namely, such a solution is hyperbolic (parabolic, elliptic, resp.) if $\kappa_{\min }<0(=0,>0$, resp.). The table below summarizes this taking into account the range of $E$ $\left(\kappa_{\max }\right.$ denotes the maximum value of $\left.\kappa\right)$.

\begin{tabular}{|l|c|c|c|}
\hline type of solution & elliptic & parabolic & hyperbolic \\
\hline range of $E$ & $-4 / 3<E<0$ & $E=0$ & $0<E<4 / 3$ \\
\hline$\kappa_{\min }$ & $>0$ & $=0$ & $<0$ \\
\hline$\kappa_{\max }$ & $>0$ & $>0$ & $>0$ \\
\hline
\end{tabular}

In any case, however, the periodic solutions can be expressed in terms of elliptic functions. More precisely, if $-4 / 3<E<4 / 3$, the solution $\kappa_{E}$ of (5.5) is given by

$$
\kappa_{E}(s)=\alpha_{3}\left(1-q^{2} \operatorname{sn}^{2}(h s, p)\right)
$$


Here,

$$
p^{2}=\frac{\alpha_{3}-\alpha_{2}}{\alpha_{3}-\alpha_{1}}, \quad q^{2}=\frac{\alpha_{3}-\alpha_{2}}{\alpha_{3}}, \quad r^{2}=\frac{1}{4}\left(\alpha_{3}-\alpha_{1}\right), \quad h=\sqrt{\frac{2}{3}} r,
$$

where $\alpha_{1}<\alpha_{2}<\alpha_{3}$ are the roots of

$$
P_{E}(y)=-2 y^{3}+6 y+3 E
$$

and $\operatorname{sn}(x, p)$ is the Jacobi elliptic sine function with modulus $p$ (see ([D]) for details).

\section{The Soliton Equations: solving for X.}

We now start the geometric and analytical descriptions of solitons. First, note that for each $-4 / 3<E<4 / 3$, the solution $\kappa$ of (5.4) is an even function with respect to its sextactic points (points where $\dot{\kappa}=0$ ). Hence, the curve $\mathbf{X}$ associated to $\kappa$ via the structure equations is invariant under the 'affine Coxeter group' generated by the affine 'reflections' corresponding to the substituitions $\mathbf{T} \rightarrow-\mathbf{T}$ and $\mathbf{N} \rightarrow \mathbf{N}$ at such points. This is, however, all that one can say about the solutions of (5.3) without using the method of adjoint orbits. So we now start to describe this technique which allows us to perform this integration.

First, notice that (5.3) and (5.4) imply that

$$
\mathbf{X}-\dot{\kappa} \mathbf{T}+\kappa \mathbf{N}
$$

is a constant vector. Hence, by translating $\mathbf{X}$, if necessary, we can assume that

$$
\mathbf{X}=\dot{\kappa} \mathbf{T}-\kappa \mathbf{N}
$$

This is a crucial reduction since now we just have to determine $g=(T, N)$ for each $\kappa$, and this is where the method of adjoint orbits comes into play. Once this has been done, (6.1) gives the parametrization for $\mathbf{X}$.

Now, for each nontrivial periodic solution $\kappa$ of (5.4) corresponding to some energy $E$ according to (5.5), consider the $s l(2, \mathbf{R})$-valued matrix

$$
\mathbf{L}=\left(\begin{array}{cc}
\dot{\kappa} \kappa & E-\frac{2}{3} \kappa^{3} \\
3-\kappa^{2} & -\dot{\kappa} \kappa
\end{array}\right)
$$


Here, $\operatorname{sl}(2, \mathbf{R})$ desnotes the Lie algebra of $S L(2, \mathbf{R})$. Recall that in view of (2.3), (2.2) now reads

$$
\mathbf{g}^{-1} \dot{\mathbf{g}}=\left(\begin{array}{cc}
0 & -\kappa \\
1 & 0
\end{array}\right) .
$$

This, together with (5.4) and (5.5), implies the validity of the Euler equations

$$
\dot{\mathbf{L}}=\left[\mathbf{L}, \mathbf{g}^{-1} \dot{\mathbf{g}}\right] .
$$

These equations mean that $\mathbf{L}$ takes values in a single adjoint orbit on $s l(2, \mathbf{R})$. In other words,

$$
\mathbf{g L g}^{-1}=\mathbf{L}_{0}
$$

where $\mathbf{L}_{0}$ is a constant matrix. This is, of course, the crucial step in the method of adjoint orbits, so we reproduce here the pertinent computation:

$$
\begin{aligned}
\frac{d}{d s}\left(\mathbf{g L g}^{-1}\right) & =\mathbf{g g}^{-1} \dot{\mathbf{g}} \mathbf{L} \mathbf{g}^{-1}+\mathbf{g} \dot{\mathbf{L}} \mathbf{g}^{-1}-\mathbf{g L g}^{-1} \dot{\mathbf{g} g} \mathbf{g}^{-1} \\
& =\mathbf{g}\left(\dot{\mathbf{L}}+\left[\mathbf{g}^{-1} \dot{\mathbf{g}}, \mathbf{L}\right]\right) \mathbf{g}^{-1}
\end{aligned}
$$

and this vanishes by (6.3).

In order to appreciate the relevance of (6.4) let us follow ([BG]) and consider, for each $\mathbf{L}_{0} \in \operatorname{sl}(2, \mathbf{R})$,

$$
\mathcal{B}_{\mathbf{L}_{0}}=\left\{(\mathbf{f}, \Lambda) \in S L(2, \mathbf{R}) \times s l(2, \mathbf{R}) ; \mathbf{f} \Lambda \mathbf{f}^{-1}=\mathbf{L}_{0}\right\} .
$$

Projection onto the second factor makes $\mathcal{B}_{\mathbf{L}_{0}}$ into a $\mathbf{G}_{\mathbf{L}_{0}}$-bundle over the adjoint orbit containing $\mathbf{L}_{0}$. Here,

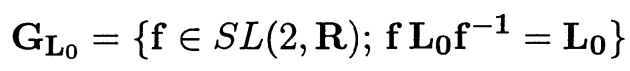

is the stabilizer subgroup of $\mathbf{L}_{0}$ relative to the adjoint action. Restriction of $\mathcal{B}_{\mathbf{L}_{0}}$ to (the image of) $\mathbf{L}$ defines a $\mathbf{G}_{\mathbf{L}_{0}}$-bundle over $\mathbf{L}$. Recalling that $\mathbf{L}$ is periodic, we can pull back this bundle under $\mathbf{L}$ to obtain a $\mathbf{G}_{\mathbf{L}_{0}}$-bundle, say $\mathcal{C}_{\mathbf{L}_{0}}$, over the unit circle $\mathbf{S}^{1}$, which happens to be isomorphic to the trivial bundle $\mathbf{S}^{1} \times \mathbf{G}_{\mathbf{L}_{0}}$. Clearly, the pair $(\mathbf{g}, \mathbf{L})$ defines a (possibly multi-valued) section of $\mathcal{C}_{\mathbf{L}_{0}}$. Now, let $(\mathbf{h}, \mathbf{L})$ represent any (single-valued) section of $\mathcal{C}_{\mathbf{L}_{0}}$. Since

$$
\mathbf{h L h}^{-1}=\mathbf{L}_{0}
$$


it follows that

$$
\mathbf{g}=\mathbf{a h},
$$

where a takes values in $\mathbf{G}_{\mathbf{L}_{0}}$. In other words, a defines a (possibly multivalued) section of $\mathcal{C}_{\mathbf{L}_{0}}$. A further computation using (6.7) shows that

$$
\mathbf{a}^{-1} \dot{\mathbf{a}}=\mathbf{h}\left(\begin{array}{cc}
0 & -\kappa \\
1 & 0
\end{array}\right) \mathbf{h}^{-1}-\dot{\mathbf{h}}^{-1} .
$$

Note that the lefthand side of this equation may be regarded as known. Hence, this defines an equation on $\mathbf{G}_{\mathbf{L}_{0}}$ satisfied by $\mathbf{a}$.

At this stage, the crucial observation is that, for $\mathbf{L}_{0} \neq 0, G_{\mathbf{L}_{0}}$ is always a one-dimensional, hence abelian, subgroup of $S L(2, \mathbf{R})$ and we can at once conclude that a can be recovered from (6.8) by a single quadrature $([\mathrm{Br}])$. This information, together with (6.7), says that the same holds for $\mathbf{g}$, and then for $\mathbf{X}$ by (6.1).

One can even go one step further and use the above setup to draw conclusions on the qualitative behaviour of $\mathbf{g}$ without explicitly integrating (5.3). First, notice that $\operatorname{det} \mathbf{L}_{0}=\operatorname{det} \mathbf{L}=-3 E$. It is not hard to check that in the parabolic or hyperbolic cases, i.e., when $\operatorname{det} \mathbf{L}_{0} \leq 0, \mathbf{G}_{\mathbf{L}_{0}}$ is isomorphic to $\mathbf{R}^{+}$, the group of positive real numbers under multiplication, so that $\mathcal{C}_{\mathbf{L}_{0}}$ is simply the cylinder $\mathbf{S}^{\mathbf{1}} \times \mathbf{R}^{+}$. Now, it is an easy matter to infer from (6.8) that $\mathbf{a}$ is defined for all values of $s$ and traces out a curve in $\mathcal{C}_{\mathbf{L}_{0}}$ with velocity bounded away from zero. It follows in particular that a never closes. Using (6.7), we conclude that $\mathbf{g}$ (and hence $\mathbf{X}$ ) never closes. In particular, $\mathbf{X}$ never defines a soliton in this range of $E$. We remark that these curves are always unbounded (see fig 3 ).

We are thus left with the elliptic range $-4 / 3<E<0$. Here, $\operatorname{det} \mathbf{L}_{0}>0$ and $\mathbf{G}_{\mathbf{L}_{0}}$ is isomorphic to $\mathbf{S}^{\mathbf{1}}$, so that $\mathcal{C}_{\mathbf{L}_{0}}$ is a torus. Applying the argument above to this case, we conclude that $\mathbf{h}$ and $\mathbf{g}$ define quasiperiodic motions on this torus. We are thus led to expect that the corresponding curves $\mathbf{X}$ are always quasiperiodic and that there exist some rationality condition that singles out the closed ones. All of these claims are confirmed by implementing the above scheme in this case. This will enable one to integrate the soliton equations as promised.

In order to proceed we shall need one more reduction, namely, if one replaces $\mathbf{g}$ in (6.4) by $\mathbf{c g}$ (which amounts to composing $\mathrm{X}$ with an affine motion) $\mathbf{L}_{0}$ gets replaced by $\mathbf{c L}_{0} \mathbf{c}^{-1}$ and this allows us to move around $\mathbf{L}_{0}$ in its adjoint orbit so that is has the simplest form. This freedom of choosing 
a suitable canonical form for $\mathbf{L}_{0}$ will be important in the computation that follows.

Since we are now in the elliptic range, set $3 E=-c^{2}$ for some $0<c<2$. We observe that the canonical form for $\mathbf{L}_{0}$ in its adjoint orbit is

$$
\mathbf{L}_{0}=\left(\begin{array}{cc}
0 & -c \\
c & 0
\end{array}\right)
$$

Now a solution in $\mathbf{h}$ for (6.6) is easily found to be

$$
\mathbf{h}=\frac{1}{\sqrt{c\left(3-\kappa^{2}\right)}}\left(\begin{array}{cc}
3-\kappa^{2} & -\dot{\kappa} \kappa \\
0 & c
\end{array}\right)
$$

and a laborious calculation using (6.8) yields

$$
\mathbf{a}^{-1} \dot{\mathbf{a}}=\frac{c}{3-\kappa^{2}}\left(\begin{array}{cc}
0 & -1 \\
1 & 0
\end{array}\right) .
$$

From this we conclude that

$$
\mathbf{a}=\left(\begin{array}{cc}
\cos \theta & -\sin \theta \\
\sin \theta & \cos \theta
\end{array}\right), \quad \dot{\theta}=\frac{c}{3-\kappa^{2}} .
$$

Coupling this with (6.7) and (6.1), the outcome of our computation is summarized as follows:

Proposition 6.1. If $\kappa$ is an elliptic solution of (5.4) then, up to affine motions, $\mathbf{X}$ takes the form

$$
\mathbf{X}=\frac{1}{\sqrt{c\left(3-\kappa^{2}\right)}}\left(\begin{array}{l}
3 \dot{\kappa} \cos \theta+c \kappa \sin \theta \\
3 \dot{\kappa} \sin \theta-c \kappa \cos \theta
\end{array}\right)
$$

where $\theta: \mathbf{R} \rightarrow \mathbf{R}$ is some (any) solution of

$$
\dot{\theta}=\frac{c}{3-\kappa^{2}} .
$$

In particular, $\mathbf{X}$ is always bounded and quasi-periodic, and it closes up (hence defining a soliton) if and only if the integral

$$
\tau(c)=\int_{\kappa_{-}(c)}^{\kappa_{+}(c)} \frac{2 \sqrt{3} c d \kappa}{\left(3-\kappa^{2}\right) \sqrt{-c^{2}+6 \kappa-2 \kappa^{3}}}
$$

is a rational multiple of $2 \pi$. Here, $0<\kappa_{-}(c)<1<\kappa_{+}(c)<\sqrt{3}$ are the two positive roots of $2 \kappa^{3}-6 \kappa+c^{2}=0$. 
The above proposition is crucial in understanding the qualitative behaviour of solitons, so we now explore its consequences.

First, if in this elliptic range, we write $\mathbf{X}$ in polar notation

$$
\mathbf{X}(s)=r(s)\left(\begin{array}{c}
\cos \phi(s) \\
\sin \phi(s)
\end{array}\right)
$$

then a computation yields

$$
\dot{\phi}=\frac{c \kappa}{6 \kappa-c^{2}}=\frac{c \kappa}{3 \dot{\kappa}^{2}+2 \kappa^{3}},
$$

so it follows that $\dot{\phi}>0$ always. Thus, the curve $\mathbf{X}$ turns steadily counterclockwise about the origin. Also note that $r^{2}=\left(6 \kappa-c^{2}\right) / c$ so $r$ reaches its maximum (resp., minimum) exactly where $\kappa$ reaches its maximum (resp., minimum).

Furthermore, the limiting behavior of $\tau(c)$ as $c$ approaches 0 from above or 2 from below is relevant to understanding which closed curves are possible, since $\tau(c)$ gives the angular displacement of $\mathrm{X}$ when $\kappa$ and $\dot{\kappa}$ undergo a complete period. Now, it follows from elementary (but tedious) computations using elliptic integrals that $\tau$ is continuous and increasing on the interval $0<c<2$ and extends continuously to the extremals of this interval if one defines $\tau(0)=\pi$ and $\tau(2)=\pi \sqrt{2}$. Thus, for any rational number $p / q$ satisfying $1 / 2<p / q<1 / \sqrt{2}$ we have $\tau(c)=(p / q) 2 \pi$, and the proposition above implies that the corresponding $\mathbf{X}$ defines a soliton. It is clear that $\mathbf{X}$ will close in $q$ complete cycles of $\kappa$ and will have $2 q$ sextactic points, half of which are maxima and half of which are minima. Moreover, $\mathbf{X}$ will have winding number equal to $p$. In particular, ellipses are the only embedded solitons.

This completes the classification of solitons in affine geometry.

Remark 6.1. Usually the denomination 'Euler equations' is reserved for equations like (6.3) arising when $\mathbf{g}$ is a solution to a homogeneous variational problem in a Lie group equipped with some left-invariant metric([B],[BG]). In this case, the Euler-Lagrange equations imply the Euler equations. Now, as can be easily checked, our problem lacks this variational structure. In spite of this we have decided to maintain the terminology essentially because the above choice for $\mathbf{L}$ has been very much modelled on the variational theory.

Remark 6.2. B. Andrews' work ([A2]) mentioned in Remark 1.4 above implies in particular that curves close enough to an ellipse converge to an 
ellipse under (3.10). A natural question here is whether the ellipse is the only soliton that is stable in this sense. If this is the case, it would be interesting to estimate the dimension of the unstable manifolds of the flow around unstable soliton solutions.

Remark 6.3. Motivated by questions in computer vision and image processing, some authors ([COT], [OST], [SP], [AST]) have considered the socalled affine invariant flow

$$
\mathbf{X}_{t}=\mathbf{N} \text {. }
$$

It can be shown (see equation (7.7) in the Appendix) that, up to inessential reparametrizations, this is equivalent to the flow

$$
\mathbf{X}_{t}=\eta^{1 / 3} \mathbf{n}
$$

where $\eta$ and $\mathbf{n}$ denote the euclidean curvature and normal, respectively. It follows that the affine invariant flow is second order in the sense that the evolution equation for $k$ is a second order parabolic equation. Moreover, this flow has a variational structure in the sense that the area enclosed by curves evolving according to it decreases as fast as possible. By a straightforward adaptation of the renormalization procedure described above, it is easy to check that ellipses are the only solitons (embedded or not) for the affine invariant flow.

\section{Appendix.}

The purpose of this appendix is to show that soliton solutions as introduced in Definition 5.1 correspond precisely to homothetic solutions of (3.10), i.e., solutions which evolve by dilation around some point.

Let us introduce some additional notation. Denote by $u$ the euclidean arclengh of $\mathbf{X}$ and by $s$ its affine arclength. Thus we have $d s=\eta^{1 / 3} d u$, where $\eta$ is the euclidean curvature, and

$$
\mathbf{T}=r^{1 / 3} \mathbf{t}
$$

where $r=\eta^{-1}$ is the curvature radius and $\mathbf{t}$ is the euclidean unit tangent vector to $\mathbf{X}$. Recall that the support function of $\mathbf{X}$ is defined by $\rho=-\langle\mathbf{X}, \mathbf{n}\rangle$, where $\mathbf{n}$ is the euclidean normal to $\mathbf{X}$ and $\langle$,$\rangle denotes the euclidean inner$ product. Let $\theta$ be the usual angular coordinate which is globally defined over $\mathbf{X}$ since $\mathbf{X}$ is locally convex. Since $\eta=d \theta / d u$, the Serret-Frenet equations now read

$$
\mathbf{X}_{\theta}=r \mathbf{t}, \quad \mathbf{t}_{\theta}=\mathbf{n}, \quad \mathbf{n}_{\theta}=-\mathbf{t}
$$


From this we get $\rho_{\theta}=\langle\mathbf{X}, \mathbf{t}\rangle$ so that

$$
\mathbf{X}=\rho_{\theta} \mathbf{t}-\rho \mathbf{n} .
$$

The question now is whether there exists a similar formula in Affine Geometry. This is the case indeed as we shall see.

Let us introduce the affine support function $\sigma=|\mathbf{X}, \mathbf{T}|$. This can be written in purely euclidean terms:

$$
\sigma=\left|\rho_{\theta} \mathbf{t}-\rho \mathbf{n}, r^{1 / 3} \mathbf{t}\right|=r^{1 / 3} \rho .
$$

The relevance of $\sigma$ is that the area enclosed by the curve is given by

$$
A(\mathbf{X})=\frac{1}{2} \int_{\mathbf{X}} \rho d u=\frac{1}{2} \int_{\mathbf{X}} \sigma d s
$$

and starting from this it is not hard to show that if $\mathbf{X}$ evolves under (4.4) then

$$
\frac{d A(\mathbf{X})}{d \tau}=\int_{\mathbf{X}} \kappa d s .
$$

It is also easy to check that

$$
\mathbf{N}=\left(r^{1 / 3}\right)_{u} r^{1 / 3} \mathbf{t}+r^{-1 / 3} \mathbf{n} .
$$

Now we compute

$$
\sigma_{s} \mathbf{T}=\left(\rho_{\theta}+\rho r^{2 / 3}\left(r^{1 / 3}\right)_{u}\right) \mathbf{t}
$$

and

$$
\sigma \mathbf{N}=\rho r^{2 / 3}\left(r^{1 / 3}\right)_{u} \mathbf{t}+\rho \mathbf{n} .
$$

It follows from (7.3), (7.8) and (7.9) that

$$
\mathbf{X}=\sigma_{s} \mathbf{T}-\sigma \mathbf{N},
$$

as desired.

After these preliminaries, we claim that soliton solutions are precisely those curves for which $\sigma=\kappa$. Recall that solitons are defined as curves $\mathbf{X}$ satisfying the structure equations

$$
\mathbf{X}_{s}=\mathbf{T}, \quad \mathbf{T}_{s}=\mathbf{N}, \quad \mathbf{N}_{s}=-\kappa \mathbf{T},
$$


where $\kappa$ is a periodic solution to

$$
\kappa_{s s}+\kappa^{2}-1=0 .
$$

Now if $\sigma=\kappa$ we find from (7.11) that $\kappa_{s}=|\mathbf{X}, \mathbf{N}|$ and $\kappa_{s s}=|\mathbf{T}, \mathbf{N}|+$ $|\mathbf{X},-\kappa \mathbf{T}|=1-\kappa \sigma=1-\kappa^{2}$ and (7.12) is satisfied. Reciprocally, if $\kappa$ is a solution to (7.12) then arguing as in Section 6 (see equation (6.1)) we find that, after possibly translating the curve, $\mathbf{X}=\kappa_{s} \mathbf{T}-\kappa \mathbf{N}$ and hence $\sigma=\kappa$.

We are thus left with the problem of checking that homothetic solutions are precisely those solutions of (3.10) such that, for each time, the curves in the evolution have the property that $\kappa$ is proportional to $\sigma$. It is convenient here to rescale the flow in such a way that the expanding curves enclose the same area, say $\pi$. If $\mathbf{X}^{(\tau)}$ denotes the one-parameter family of evolving curves, we define

$$
\tilde{\mathbf{X}}^{(\tilde{\tau})}=\sqrt{\frac{\pi}{A\left(\mathbf{X}^{(\tau)}\right)}} \mathbf{X}^{(\tau)}
$$

and compute the evolution of $\tilde{\mathbf{X}}$ with respect to the rescaled time

$$
\tilde{\tau}(\tau)=\int_{0}^{\tau}\left(\frac{\pi}{A\left(\mathbf{X}^{(\tau)}\right)}\right)^{4 / 3} d \tau .
$$

Using (7.13), (7.14) and (7.6), we get

$$
\tilde{\mathbf{X}}_{\tilde{\tau}}=-\tilde{\kappa} \tilde{\mathbf{N}}-\tilde{\Lambda} \tilde{\mathbf{X}},
$$

where a tilde refers to those invariants associated to the rescaled curves and.

$$
\tilde{\Lambda}=\frac{1}{2 \pi} \int_{\tilde{\mathbf{X}}} \tilde{\kappa} d \tilde{s}
$$

Inserting (7.10) into (7.15) we obtain the evolution equation for the rescaled. flow:

$$
\tilde{\mathbf{X}}_{\tilde{\tau}}=(-\tilde{\kappa}+\tilde{\Lambda} \tilde{\sigma}) \tilde{\mathbf{N}}-\tilde{\Lambda} \tilde{\sigma}_{\tilde{s}} \tilde{\mathbf{T}} .
$$

We now appeal once again to the well-known principle that tangential terms do not affect the geometry of the evolution in order to conclude that the flow (7.16) is geometrically equivalent to the flow

$$
\tilde{\mathbf{X}}_{\tilde{\tau}}=(-\tilde{\kappa}+\tilde{\Lambda} \tilde{\sigma}) \tilde{\mathbf{N}},
$$

and this clearly implies that a curve expands by homotheties about some point (i.e. $\tilde{\mathbf{X}}_{\tilde{\tau}}=0$ ) if and only if the affine curvature is proportional to the affine support function (i.e. $\tilde{\kappa}=\tilde{\Lambda} \tilde{\sigma}$ ), as desired. 

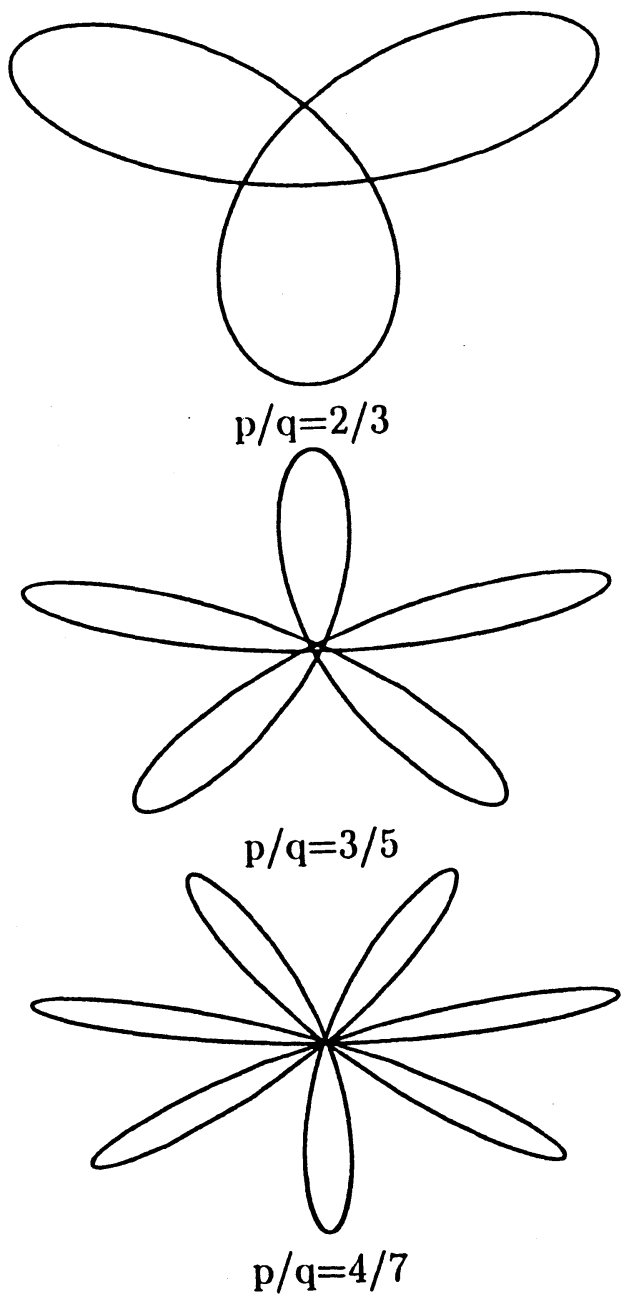

Figure 2: Closed Curves 


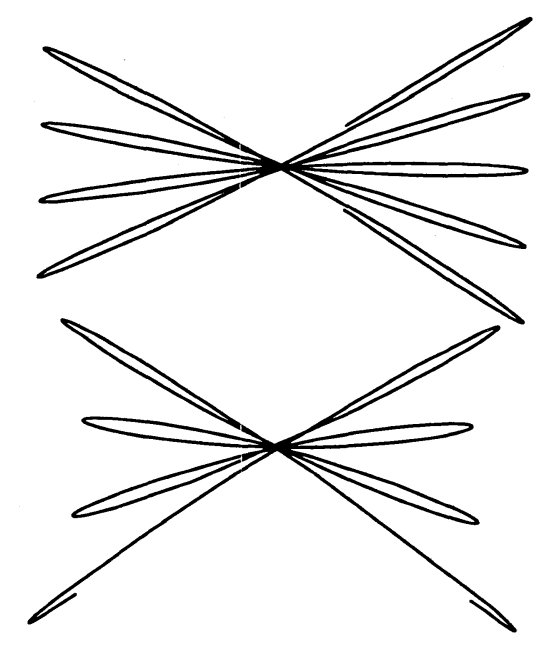

Figure 3.

\section{References.}

[AL] U. Abresch and J. Langer, The normalized curve shortening flow and homothetic solutions, J. of Diff. Geom. 23 (1986), 175-196.

[A1] B. Andrews, Evolving convex curves, SMS preprint 1997.

[A2] B. Andrews, The affine curve-lengthening flow, SMS preprint, 1997.

[AST] S. Angenent, G. Sapiro and A. Tannenbaum, On the affine invariant heat equation for non-convex curves, preprint.

[Bl] W. Blaschke, Vorlesung uber Differentialgeometrie, Springer, Berlin, 1923.

[Br] R. Bryant, An introduction to Lie groups and symplectic geometry IAS/Park City Series, Vol. 1, D. S. Freed, K. K. Uhlenbeck, editors.

[BG] R. Bryant and P. Griffiths, Reduction for constrained variational problems and $\int \kappa^{2} / 2 d s$, Amer. J. of Math. 108 (1986), 525-570.

[COT] E. Calabi, P. Olver, P. and A. Tannenbaum, Affine geometry, curve flows and invariant numerical approximations, Adv. in Math. 124 (1996), 154196.

[D] H. Davis, Introduction to nonlinear differential and integral equations, Dover, New York, 1962. 
[GH] M. Gage and R. Hamilton, The shrinking of convex curves by the heat equation, J. of Diff. Geom. 23 (1986), 69-86.

[GL] M. Gage and Y. Li, Evolving plane curves by curvature in relative geometries, Duke Math. J. 75 (1994), 79-98.

[G] M. Grayson, The heat equation shrinks embedded plane curves to round points, J. of Diff. Geom. 26 (1987), 285-314.

[LS] J. Langer and D. Singer, The total squared curvature of closed curves, J. of Diff. Geom. 20 (1984), 1-22.

[L] A. Linnér, Curve-straightening, Proc. of Symp. Pure Math., vol. 54, part 3, 451-458.

[OST] P. Olver, G. Sapiro and A. Tannenbaum, Affine invariant gradient flows, preprint.

[SP] G. Sapiro and A. Tannenbaum, On affine plane curve evolution, J. of Func. Anal. 119 (1994), 79-120.

ReCeived SePtember 19, 1997.

Departamento de Matemática

Universidade Federal do Ceará

FORTALEZA - BRASIL

E-mail addresses: levi@mat.ufc.br

fabio@mat.ufc.br 